\section{Orchestrating metastasis}

It is becoming increasingly clear that cancer cells play an active part in stimulating bone-marrow-derived cells (BMDCs) to create a microenvironment that is favourable for metastasis (the metastatic niche). However, the mechanisms controlling this process are not well understood. Michael Karin and colleagues have uncovered a new pathway between cancer cells and BMDCs that leads to increased metastasis.

Kim et al. analysed four cancer cell lines and found that the ability of conditioned media from metastatic cells to stimulate production of interleukin 6 (IL-6) and tumour necrosis factor- $\alpha$ (TNF $\alpha)$ in bone-marrowderived macrophages (BMDMs) was greater than that of conditioned media from non-metastatic cells. Specifically, metastatic Lewis lung carcinoma (LLC) cell conditioned medium (LCM) induced high levels of IL- 6 and TNF $\alpha$ in BMDMs. Tnfa ${ }^{-/-}$ mice, but not $\mathrm{Il}^{-/-}$mice, injected with LLC cells had significantly improved survival and reduced lung tumour multiplicity compared with injected wild-type mice, indicating that $\mathrm{TNF} \alpha$ is crucial for LLC metastasis.

$\mathrm{TNFa}$ is induced through the stimulation of Toll-like receptors (TLRs), and the authors found that BMDMs lacking Tlr 2 could not be activated by LCM. Furthermore, inflammation, Tnfa mRNA and micrometastases were reduced in the lungs of $T l r 2^{-/-}$mice injected through the tail vein with LLC cells. Subcutaneous implantation of LLC cells also resulted in fewer lung and liver tumour nodules and adrenal gland metastases in $\mathrm{Tlr} 2^{-/-}$mice, but no differences were observed in primary tumour growth. Wild-type mice reconstituted with $T l r 2^{-/-}$bone survival after LLC injection compared with mice reconstituted with wild-type bone marrow (WT/WT), indicating that TLR2 in BMDCs is crucial for metastasis. WT/WT mice, but not WT/Tlr2 $2^{-/-}$mice, had more metastases when injected with both LLC cells and LCM than when injected with LLC cells alone, suggesting that factors secreted by LLC cells can activate TLR2 in BMDCs and enhance metastasis.

What factors from LLC cells stimulate TLR2? The authors collected LCM in vitro and, using protein purification columns, isolated several extracellular matrix peptides that could stimulate BMDMs, including versican $\mathrm{V} 1$, laminin- $\beta 1$, thrombospondin 2 and procollagen III-al. Experiments with neutralizing antibodies and short hairpin RNAs in LLC cells indicated that versican V1 from LCM could enhance TNFa production and metastasis. Importantly, silencing of versican V1 in LLC cells significantly improved the survival of mice injected with these cells and reduced metastases (with little or no effect on primary tumour growth) in the subcutaneous implantation model. Stimulation of wild-type and $T l r 2^{-/-}$BMDMs with purified versican $\mathrm{V} 1$ suggested that versican V1 acts through TLR2, although it is not clear whether the two proteins interact directly. marrow (WT/Tlr2 ${ }^{-/-}$) had improved
Versican is frequently upregulated in non-small-cell lung cancer, so the versican-TLR2-TNFa pathway in cancer cells and BMDMs offers several possible targets for therapeutic intervention and inhibition of metastasis.

Sarah Seton-Rogers

ORIGINAL RESEARCH PAPER Kim, S. et al.

Carcinoma-produced factors activate myeloid cells through TLR2 to stimulate metastasis. Nature 457, 102-106 (2009)

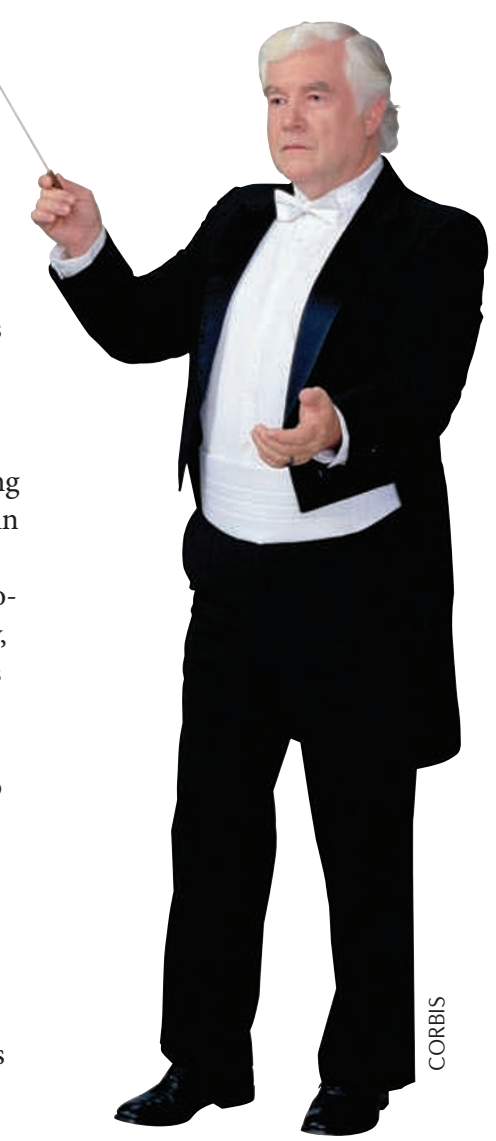

